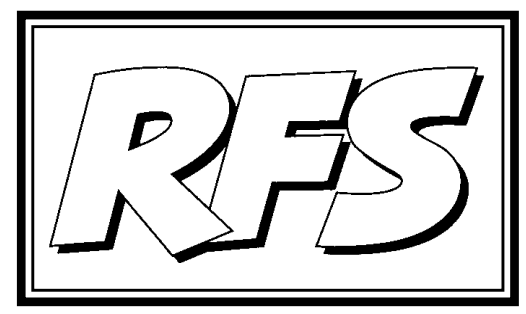

Revista de Fomento Social, 60 (2005), 189-219

\title{
El pensamiento social de Juan Pablo II. Líneas básicas de sus tres encíclicas sociales
}

\section{Ildefonso CAMACHO LARAÑA S .J. ${ }^{1}$}

(Palabras clave: Doctrina Soctal de la IGLESIA, Encíclicas.

KEY WORDS: SOCIAL DOCTRINE OF THE CHURCH, ENCYCLICALS)

Los 26 años y medio de pontificado de Juan Pablo II se corresponden con un periodo muy cargado de acontecimientos, algunos de los cuales han marcado la historia contemporánea: concretamente, la caída del muro de Berlín como símbolo del fracaso del colectivismo y la nueva configuración del mundo que va resultando de ello. El pensamiento social del pontífice

\footnotetext{
1 Facultad de Teología. Granada.
} 
recientemente desaparecido debe ser leído desde ese trasfondo para valorar mejor algunos rasgos esenciales de la Doctrina Social de la Iglesia: nos referimos a su inevitable condición histórica, en dependencia de los acontecimientos que la rodean.

Es evidente que el término empleado (doctrina) más bien induce a pensar en un sistema cerrado de principios. Por eso es preciso salir al paso de este equívoco, muchas veces abonado desde determinadas posiciones ideológicas, que buscan ante todo la seguridad en la doctrina. Pero el pensamiento social de la Iglesia se ha ido elaborando en la época moderna de forma progresiva, de manera que se detecta en él el esfuerzo por mantener un equilibrio entre principios inalterables y aplicaciones prácticas ${ }^{2}$. Es más, no siempre es posible hacer una separación tajante entre aquéllos y éstas: sería preferible hablar de una permanente remodelación de los temas, donde uno puede detectar que hay principios de fondo recurrentes, pero que éstos no bastan para iluminar los problemas concretos. Es ahí donde estriba, en nuestra opinión, el principal valor de la Doctrina Social de la Iglesia. Pero no basta con reconocer ese valor; es necesario además tenerlo muy en cuenta a la hora de leer e interpretar los documentos.

Juan Pablo II es, como decíamos, una expresión muy ilustrativa de este modo de proceder. Para mostrarlo vamos a tomar como base sus tres grandes encíclicas sociales: primero haremos una presentación muy esquemática de cada una de ellas, subrayando lo que en ellas hay de diverso; después analizaremos algunos puntos de convergencia de las tres; por último, quisiéramos también dejar constancia de algunas aportaciones más significativas de Juan Pablo II a la Doctrina Social de la Iglesia en relación con los desarrollos anteriores a él.

Hemos optado por limitarnos a sus tres encíclicas sociales: Laborem exercens (1981), sobre el trabajo humano; Sollicitudo rei socialis (1987), sobre las diferencias mundiales y el conflicto entre "hiperdesarrollo" de unos pueblos y subdesarrollo de otros; Centesimus annus $(1991)^{3}$, sobre el centenario de la

2 Así se ha reconocido, de forma autorizada, recientemente: "La Doctrina Social de la Iglesia no ha sido pensada desde el principio como un sistema orgánico, sino que se ha formado en el curso del tiempo, a través de las numerosas intervenciones del Magisterio sobre temas sociales". Pontificio Consejo Justicia y Paz (2005), Compendio de la Doctrina Social de la Iglesia, Roma, Libreria Editrice Vaticana, n. 72.

3 En adelante estas tres encíclicas serán citadas por sus siglas: LE, SRS, CA. 
primera gran encíclica social, escrita por León XIII, pero con una profunda reflexión sobre la caída del colectivismo. Ellas representan lo esencial de su pensamiento, expresado además en multitud de intervenciones para las más diversas circunstancias.

Comenzaremos con una breve presentación de esos tres grandes documentos sociales. Ellos encierran una gran riqueza de pensamiento y abordan innumerables cuestiones. Pretendemos ceñirnos a lo esencial, que es donde radica su valor principal. Con ello evitaremos dos peligros en que frecuentemente caen quienes utilizan estas encíclicas: por una parte, un excesivo deseo de emplearlas para apoyar determinadas posturas previas de quien los utiliza; por otra, la cita fragmentaria y descontextualizada de algunos pasajes, que impide comprender su verdadero alcance, cuando no las malinterpreta.

Entendemos por lo esencial: el contexto de la encíclica y los problemas a los que pretende responder; el tema central y el objetivo de la misma; el esquema básico de su desarrollo; los temas más nucleares que contiene.

\section{Encíclica sobre el trabajo humano: Laborem exercens (1981)}

Esta encíclica, situada muy al comienzo del pontificado de Juan Pablo II, refleja su preocupación por el mundo comunista de Europa, que él acaba físicamente de abandonar, cuando todavía no existen indicios de que el colectivismo va a sucumbir. Es un dato que debe presidir todo estudio del documento.

\subsection{Contexto y objetivos}

Porque dicha circunstancia explica lo que pudiera parecer un cierto retroceso en la Doctrina Social respecto a los enfoques dominantes en ella desde mitad del siglo XX: en efecto, si hasta entonces había estado muy condicionada por las relaciones entre los dos grandes sistemas socioeconómicos y las ideologías que les acompañaban (situación que podríamos visualizar como el conflicto Este-Oeste), a partir de ese momento había girado hacia los grandes problemas mundiales y las diferencias crecientes entre países desarrollados y países en desarrollo (el conflicto Norte-Sur). LE parece volver al escenario del conflicto Este-Oeste. Ésa era la cuestión social en el mundo industrial; pero ya convenía hablar de cuestión social mundial. ¿Influye en 
esta cierta regresión el hecho de que aquél seguía siendo todavía el gran problema de la Europa comunista?

Pero este retorno a enfoques anteriores no debe impedir que reconozcamos la novedad de LE. Ésta radica precisamente en la perspectiva que se adopta para analizar toda la cuestión social: el trabajo humano. En realidad, el trabajo ocupa un lugar central en los documentos de finales del siglo XIX y primera mitad del XX, como efecto del planteamiento común de toda esa época: el de la confrontación capitalismo-socialismo (y capitalismo-colectivismo desde la revolución rusa de 1917 y, más aún, desde el final de la segunda guerra mundial), que deriva siempre en las relaciones conflictivas capital-trabajo. Ahora bien, dado que el régimen de propiedad era el rasgo que distinguía entre sí a los dos sistemas económicos, la doctrina sobre la propiedad era el primer centro de atención en todos los documentos. Sólo una vez asentado el principio de la propiedad privada y los criterios éticos correspondientes, se abordaba el tema del trabajo.

Posteriormente este orden se invertirá: ya la constitución pastoral Gaudium et spes del Vaticano II tratará en primer lugar del trabajo, expresando así la prioridad de éste sobre la propiedad. LE irá más lejos: enfocará toda la doctrina sobre la propiedad como una consecuencia de la visión que ofrece del trabajo humano. No es sólo un cambio en el orden de tratamiento: asistimos ahora a toda una relectura de la propiedad desde el trabajo.

\subsection{Contenido esencial}

Con este enfoque como base, el contenido esencial puede esquematizarse como sigue:

I. La doctrina [Capítulo 2: "El trabajo y el hombre"]:

1. El trabajo: visión teológica y antropológica. El punto de partida es bíblico y teológico: el texto de la creación ("Llenad la tierra y sometedla", Gen 1,28). La referencia antropológica parte de la consideración del trabajo como actividad transitiva (y no inmanente a la persona).

2. Doble consideración del trabajo. Su dimensión objetiva y su dimensión subjetiva. La primera se refiere a lo que hay en cada trabajo de específico y tiene que ver con el producto concreto de cada uno de ellos; el segundo se refiere a lo que es común a todo trabajo: que 
hay una persona humana detrás de él. Si en el trabajo objetivo se expresa el dominio del ser humano sobre el mundo, en el trabajo subjetivo se expresa la persona humana como imagen de Dios.

3. Un principio ético derivado: la prioridad del trabajo subjetivo sobre el trabajo objetivo. Porque lo que verdaderamente dignifica el trabajo es el hecho de que lo hace una persona humana. Este principio es el eje de toda la encíclica: más aún, es la clave desde la que debe analizarse toda la cuestión social.

II. La aplicación de dicha doctrina [Capítulo 3: "Conflicto entre trabajo y capital en la presente fase histórica"]:

1. En la sociedad industrial moderna se da un conflicto entre capital y trabajo, que aún perdura.

2. Este conflicto, que se agudizó con el capitalismo, tampoco fue resuelto por el colectivismo, aunque se propusiera hacerlo. Y es que ambos sistemas socioeconómicos han invertido el principio anterior, dando prioridad al capital sobre el trabajo. Porque uno y otro han caído en el mismo doble error: economicismo (el valor del ser humano se mide por sus resultados económicos) y materialismo (prioridad de lo material).

3. La solución a ese conflicto tiene que venir de la aplicación del principio antes enunciado, que ahora se traduce como prioridad del trabajo sobre el capital.

4. Consecuencias para la doctrina sobre la propiedad, tema siempre debatido en la confrontación entre los sistemas socioeconómicos modernos: lo decisivo no es que la propiedad sea pública o privada, sino que esté al servicio del trabajo.

5. Esto significa que, tanto en un sistema como en otro, el trabajador ha de ser verdaderamente sujeto de la actividad económica, es decir, ha de ser participe en el trabajo que realice como ser humano (con todas sus potencialidades) y no sólo como máquina.

\section{Temas complementarios:}

1. Los derechos inherentes al trabajo [Capítulo 4: "Derechos de los hombres del trabajo"]. De la dignidad del trabajo derivan ciertos derechos: al trabajo mismo, al salario y a otras prestaciones sociales. Igualmente se requiere un tratamiento digno de todo ser humano 
para situaciones particulares: trabajador agrícola, inmigrante, minusválidos, etc.

2. El Evangelio del trabajo [Capítulo 5: "Elementos para una espiritualidad del trabajo"]. La Iglesia ofrece la "buena nueva" sobre el trabajo, que descubre su sentido último: el ser humano, creado a imagen y semejanza de Dios, participa en su obra creadora; el ejemplo de Jesús como hijo de trabajador; el trabajo como fatiga recibe su sentido último de la muerte y la resurrección de Cristo.

\subsection{Puntos a destacar y principales aportaciones}

Tras este esquema del contenido, podemos todavía destacar algunos aspectos de la encíclica:

a) Como cuestión previa conviene señalar que la encíclica es de difícil lectura y comprensión. No basta una lectura rápida para hacerse cargo de su contenido. El lenguaje es, con frecuencia, oscuro. Las repeticiones son numerosas. Aunque el esquema general de la encíclica es nítido, el desarrollo de las ideas dentro de cada capítulo no es siempre lineal, lo que puede producir cierta desorientación en el lector.

b) En el documento hay una notable preocupación por mantener un doble nivel de discurso: uno, de carácter filosófico y antropológico; otro de carácter teológico y cristiano. Así se constata desde el principio cuando se presenta la doctrina sobre el trabajo. Y a ello obedecen también los dos últimos capítulos: el cuarto, con consideraciones ético-filosóficas sobre los derechos del trabajo; el quinto, con una reflexión cristiana sobre la espiritualidad del trabajo.

c) Una novedad muy llamativa en esta encíclica, en contraste con toda la Doctrina Social anterior, es el tratamiento simétrico que se hace de los dos sistemas socioeconómicos ${ }^{4}$. En efecto, hasta Juan Pablo II se

4 Cuando Juan Pablo II habla en LE del sistema alternativo al capitalismo, siempre se refiere al colectivismo en la forma concreta en que se desarrollaba entonces en la Europa comunista, de la que él procedía. Esta circunstancia debe ser muy tenida en cuenta a la hora de comparar estos textos con los de pontífices anteriores: por ejemplo, con los de León XIII en Rerum novarum (que se refieren al socialismo revolucionario de finales del siglo XIX) o con los de Pablo VI en Octogesima adveniens (que están pensados para las diversas formas del socialismo en la segunda mitad del siglo XX, marxistas o socialdemócratas). 
había abundado en la diferencia entre los dos sistemas, consistente en el distinto tratamiento dado al régimen de propiedad: la opción por la propiedad privada (muy radical al comienzo, más matizada ya a mediados del siglo XX) situaba a la Doctrina Social de la Iglesia más cerca del capitalismo que del colectivismo. Ahora Juan Pablo II, sin ignorar esta diferencia, considera más relevante para su análisis aquello en que ambos coinciden: el no haber sabido hacer realidad la prioridad del trabajo sobre el capital.

d) En el tratamiento de estos sistemas se impone un cierto pragmatismo, pero a la vez se advierte ya lo que será un enfoque muy característico en el pensamiento social de Juan Pablo II. Después de hacer la crítica de los dos sistemas, el Papa propone vías para una posible corrección de ambos, que también coinciden: la participación efectiva del trabajador en la actividad productiva, dando a este término de "participación" un sentido lo más amplio posible, y siempre buscando superar que el trabajador tenga que limitarse a realizar órdenes que le vienen de arriba, sin llegar a poner nunca en juego sus capacidades específicamente humanas. En eso es manifiesto su realismo pragmático que huye de descalificaciones globales. Pero es de destacar también que la propuesta que se ofrece se sitúa no en el nivel de alternativas, sino de transformación de lo existente incorporándole valores nuevos (concretamente, la participación): para Juan Pablo II, el tema de los valores es decisivo, como tendremos ocasión de confirmar.

e) Todo esto afecta a la doctrina sobre la propiedad, a propósito de la cual encontramos formulaciones que contrastan significativamente con toda la tradición anterior. Dicha tradición comenzó con una acendrada defensa de la propiedad privada en el siglo XIX y la exclusión sin matices de la propiedad pública, en plena polémica con el socialismo revolucionario de aquel tiempo. Se matizó esta posición posteriormente, insistiendo más en la compatibilidad entre ambas formas de propiedad (Mater et magistra, Gaudium et spes). Ahora LE relativiza el hecho de que la propiedad sea privada o pública y coloca como criterio esencial el que esté al servicio del trabajo, en el sentido de que no se subordine el trabajo a los intereses de la propiedad o a la acumulación de la misma (poniendo todo el acento en la producción económica).

f) Por último, LE deja claro que la última fuente de inspiración de todo su pensamiento está en una visión cristiana del ser humano. Sólo desde 
una adecuada antropología se pueden abordar los problemas sociales de nuestro tiempo. Y esta antropología deriva de la tradición cristiana, pero también de una correcta filosofía sobre el hombre.

Como conclusión, conviene insistir en el contraste que representa LE entre novedad y tradición. Lo tradicional en ella es el marco: una sociedad industrial atenazada por el conflicto capital-trabajo, pero vivida ahora desde la experiencia directa de un país colectivista y con no pocas reservas sobre el modelo occidental capitalista. Lo nuevo es el enfoque, más inspirado por la antropología filosófica y cristiana y, sólo en un momento posterior, iluminado desde las ciencias sociales.

\section{Encíclica sobre el desarrollo de los pueblos: Sollicitudo rei socialis (1987)}

Si LE tenía como referente el conflicto capital-trabajo propio de las sociedades industriales, esta nueva encíclica adopta una perspectiva mucho más universal y toma como objeto el debate sobre el desarrollo de los pueblos y el conflicto Norte-Sur. No se olvide que estamos en el final de la "década perdida" para muchos países del tercer mundo ${ }^{5}$.

\subsection{Contexto y objetivos}

En efecto, tras la crisis de los años 1970, la aplicación de rigurosos planes de ajuste estructural, inspirados por el resurgir impetuoso del credo liberal, llevaron a estos países a trances de auténtica gravedad. Fue el durísimo precio que se pagó para lograr unos equilibrios macroeconómicos que se consideraban condición previa indispensable para reiniciar los procesos de desarrollo. Pero en los países afectados el resultado fue no sólo de estancamiento económico y aumento de la pobreza, sino de creciente desesperanza.

Todo ello contrasta con el mantenimiento de aceptables ritmos de desarrollo y bienestar en el Norte, donde la crisis no había dejado de hacer mella, pero se había encontrado con países menos vulnerables y con más recursos para resistir a sus efectos más perniciosos.

Este contraste, que constituye un capítulo más del conflicto Norte-Sur,

5 La denominación fue utilizada también por el BAnco MundiaL en su Informe sobre el desarrollo mundial 1990. La pobreza, Washington 1990, 7. 
da a Juan Pablo II la ocasión para conmemorar el vigésimo aniversario de la primera encíclica sobre el desarrollo (Populorum progressio). La había publicado Pablo VI en 1967. Abría con ella un frente inédito en la Doctrina Social de la Iglesia, que hasta entonces se había mantenido más circunscrita a los problemas de las sociedades industriales occidentales; y se colocaba en línea con las orientaciones del Concilio en su constitución sobre la Iglesia en el mundo de hoy (Gaudium et spes). Veinte años después, Juan Pablo II pretende someter a revisión toda esta problemática, consciente de que las dos décadas transcurridas aportaban nuevos datos y nuevos elementos de reflexión y permitían nuevas propuestas de acción.

Queda así de manifiesto - como decíamos al comienzo de este artículo-el carácter histórico de la Doctrina Social de la Iglesia, que permite volver sobre el mismo tema, no sólo para hacer nuevas aplicaciones a una realidad cambiante, sino también para remodelar la doctrina elaborada sobre el mismo. Éste es el sentido de la segunda encíclica social de Juan Pablo II.

\subsection{Contenido esencial}

En cuanto a su contenido esencial, el texto responde de forma bastante fiel al esquema de "ver/juzgar/actuar"6 en contraste con LE, que procedía metodológicamente de forma más deductiva (a partir de presupuestos doctrinales, bíblicos o antropológicos). El esquema puede desarrollarse asî7:

I. Ver [Capítulo 3: "Panorama del mundo contemporáneo"]: análisis de la situación

1. Los hechos: las diferencias no sólo persisten después de veinte años, sino que se han acrecentado en este tiempo que nos separa de la encíclica de Pablo VI; y, lo que es más grave, la esperanza ha desaparecido. Por otra parte, el mundo es cada vez más interdependiente.

2. La interpretación de estos hechos: el escandaloso contraste entre el "hiperdesarrollo" del Norte y el subdesarrollo del Sur es consecuen-

6 Este esquema comprende los capítulos 3 a 6 . Los tres capítulos restantes son: el 1ํㅜㄹ introductorio; el $2^{\circ}$, conmemorativo de la encíclica Populorum progressio; el $7^{\circ}$, conclusivo.

7 El esquema está tomado, casi literalmente, de mi anterior escrito: I. CAMACHO (1996), "Una mirada de conjunto a las tres encíclicas sociales de Juan Pablo II”, Proyección 43, 265-286. 
cia de la división de bloques en el Norte, dos bloques confrontados entre ellos a todos los niveles (ideológico, económico, político, militar) que generan una dinámica neoimperialista sobre el planeta, la cual priva de autonomía a los países más atrasados.

II. Juzgar: reflexión ética, desde una doble perspectiva.

1. Criterios para el juicio [Capítulo 4: "El auténtico desarrollo humano"], que son de dos tipos:

- Éticos: el verdadero desarrollo supone una adecuada subordinación del tener al ser, así como el respeto a la dimensión espiritual, la propiamente específica, del ser humano.

- Teológicos: el hombre es continuador de la obra creadora deDios, lo que da el sentido último al desarrollo; y además trabaja para que toda la creación se encamine hacia la salvación prometida en Cristo.

2. Juicio de la realidad analizada [Capítulo 5: "Una lectura teológica de los problemas modernos"]: ese mundo interdependiente y dividido, a la luz de esos criterios éticos, merece ser calificado como estructuras de pecado; las principales de ellas son el afán de ganancia y la sed de poder, ambas absolutizadas, las cuales constituyen el sistema de valores vigente en nuestro mundo.

III. Actuar: algunas propuestas

1. Hay que impulsar un sistema ético alternativo al anterior, que tenga como su eje principal la solidaridad. En efecto, ésta es la mejor respuesta a la interdependencia que caracteriza a nuestro mundo. Esta solidaridad es, a la vez, actitud moral y virtud cristiana.

2. [Capítulo 6: "Algunas orientaciones particulares"] Algunas propuestas más concretas, que sólo ese sistema ético alternativo es capaz de hacer posible: aparte del esfuerzo de los países subdesarrollados y de la colaboración de todos, se precisan una serie de reformas de los mecanismos de funcionamiento de la economía mundial y de las instituciones internacionales. 


\subsection{Puntos a destacar y principales aportaciones}

Con este panorama temático de fondo es posible ahora identificar los aspectos concretos en que SRS representa una aportación significativa a la Doctrina Social de la Iglesia.

a) Probablemente la contribución de más alcance se refiere al concepto mismo de Doctrina Social de la Iglesia. Es sabido que en el Concilio y en los documentos de Pablo VI esta expresión se eludió sistemáticamente, quizás por las contraindicaciones que señalábamos más arriba. Juan Pablo II, no sólo la retoma con decisión desde el comienzo mismo de su pontificado, sino que precisa su contenido, poniéndola en estrecha relación con la misión evangelizadora de la Iglesia. Esta relación se desdobla en dos aspectos:

- No es objeto de la Doctrina Social de la Iglesia ofrecer alternativas concretas a los sistemas socioeconómicos vigentes, aunque se muestre crítica con ellos. No se mueve en el campo de las ideologías, sino que es "la cuidada formulación del resultado de una atenta reflexión sobre las complejas realidades de la vida del hombre en la sociedad y en el contexto internacional, a la luz de la fe y de la tradición eclesial". Y continúa: "Su objetivo principal es interpretar esas realidades, examinando su conformidad o diferencia con lo que el Evangelio enseña acerca del hombre y su vocación terrena y, a la vez, trascendente, para orientar en consecuencia la conducta cristiana" [SRS 41]. Su campo es el de la teología moral.

- La Doctrina Social de la Iglesia es un instrumento para la evangelización, entendida ésta no sólo como el anuncio explícito del Evangelio, sino como una tarea profética, que incluye la denuncia de los males e injusticias y el anuncio de nuevas formas de convivencia humana ${ }^{8}$.

8 Para comprender adecuadamente lo que esto significa es preciso tener en cuenta la revisión del concepto de evangelización que hizo Pablo VI, siguiendo la reflexión del Vaticano II sobre la misión de la Iglesia. La evangelización es un "proceso complejo" donde se implica la comunidad creyente toda, que comprende fases sucesivas, como son el testimonio cristiano, el anuncio explícito, la incorporación a la comunidad creyente (para este nuevo enfoque es esencial la Exhortación apostólica Evangelii nuntiandi, que publicó PABLo VI en 1975, como resultado de Sínodo universal de obispos celebrado el año anterior). En ese primer momento del testimonio, que abre el camino para el anuncio explícito del Evangelio, se incluye el compromiso cristiano de transformación de la realidad, donde los creyentes actúan a la luz de la Doctrina Social, pero buscan llegar a concreciones mayores, que representen verdaderas alternativas para las estructuras sociales y económicas de nuestro mundo. Si la 
b) Este enfoque de la Doctrina Social explica en qué lugar se sitúa la Iglesia ante el problema de las diferencias escandalosas entre el "hiperdesarrollo" del Norte y el subdesarrollo del Sur: más allá de los sistemas socioeconómicos, de los que se hace una fuerte denuncia, la crítica más específica de la Iglesia se sitúa en los sistemas de valores que subyacen a ellos, para aportar después un sistema de valores alternativo. La alternativa no está, pues, en los sistemas de organización de la sociedad, sino en los sistemas de valores que los sustentan. ¿Cuál es el sistema vigente y cuál el alternativo?:

- El sistema vigente, que la encíclica critica designándolo como estructuras de pecado, está caracterizado por dos valores dominantes, que tienden a absolutizarse: la sed de ganancia y el afán de poder. Estos valores, que podríamos identificar con la competitividad, son los que sirven como criterios de decisión espontáneamente aplicados en cualquier situación. No sólo legitiman los sistemas socioeconómicos y políticos, sino que explican también el comportamiento de las personas.

- El sistema alternativo tiene como eje, frente a la competitividad, la solidaridad. Ésta es definida en una forma que se contrapone claramente a la competitividad: "es la determinación firme y perseverante de empeñarse por el bien común; es decir, por el bien de todos y cada uno, para que todos seamos verdaderamente responsables de todos" [SRS 38]. En efecto, este sentirnos "todos responsables de todos" implica una lógica diferente a la propia de la competitividad, donde todos vivimos en pugna con todos.

c) Pero la crítica de los sistemas económicos no falta aquí, y va en una línea que recuerda la de LE: fijándose más en sus puntos de coincidencia que en las diferencias ${ }^{9}$. Lo que denuncia Juan Pablo II como causa "geopolítica"

Doctrina oficial no desciende a esas concreciones, el cristiano no puede eludirlas uniendo a su motivación e inspiración creyente su competencia en las ciencias humanas y sociales.

9 La revista 30 Giorni dedicó un "Dossier: el Papa ante los dos imperialismos" en su número de abril de 1988 (pp. 32-42) a la mala acogida de SRS en Estados Unidos, sobre todo en los ambientes neoconservadores y neoliberales. En el se inserta un artículo de Michael Novak, en que protesta contra esta "acusación de equidistancia" de ambos sistemas. Después de analizar los diez pasajes en que SRS destacaba el "paralelismo moral" entre Este y Oeste concluía que falla en la encíclica la interpretación del liberalismo. Para él la sociedad liberal supone el control de la búsqueda del lucro económico y de toda forma de poder. Y apostillaba que los criterios morales de la encíclica eran sencillamente liberales: en lo político (democracia, soberanía de la ley, protección de los derechos), en lo económico (libre iniciativa), 
del grave retraso en el proceso de desarrollo de muchos pueblos es la contraposición de dos bloques inspirados respectivamente por el capitalismo liberal y el colectivismo marxista:

- Estos dos bloques son considerados como sistemas globales de organización de la sociedad: por eso se habla de una contraposición a varios niveles, ideológico, económico, político y militar. Esta forma de analizar los sistemas de organización de la sociedad, distinguiendo en ellos diversos niveles interrelacionados, lo reencontraremos en CA: lo ideológico es lo que da sustento, legitimación y coherencia a todo lo demás.

- Esta dinámica de enfrentamiento induce a cada uno de los bloques a ampliar el ámbito de su influencia en el mundo y hace imposible que los países más débiles y dependientes puedan emprender un proceso autónomo de desarrollo.

d) SRS mantiene, como LE, una clara distinción en los niveles de su discurso: pero no sólo subrayando la diferencia entre el discurso ético y el teológico, sino adentrándose además con cierta audacia en el análisis social, que indaga causas antes de pronunciar juicios éticos:

- La distinción del discurso ético y del teológico es muy palpable, al menos en dos temas: cuando se habla del desarrollo (como quedó de manifiesto en el esquema recogido más arriba), hay una reflexión que se basa en la antropología y otra que se adentra en la teología de la creación y de la redención; y cuando se presenta la solidaridad como eje de un nuevo sistema de valores, se indica que estamos ante una virtud moral, pero que puede ser también enriquecida desde la perspectiva cristiana (es también una virtud cristiana).

- En el análisis social se apunta como causa principal (no única) del subdesarrollo la contraposición de los dos bloques. Evidentemente éste es un diagnóstico que no todos los analistas del desarrollo compartirán, puesto que son muchos los que atribuyen el retraso de algunos países

en lo cultural (solidaridad). Cf. M. NovAK, "Pero el Papa no nos comprende", 30 Giorni (abril 1988), 37-40. Quizás habría que decirle a Novak que Juan Pablo II se estaba fijando en la realidad mientras que él se movía en el terreno de la teoría (más concretamente, en la de los clásicos del liberalismo, no siempre recogidos fielmente por los autores neoliberales ...). En España también esta crítica de neoconservadores y neoliberales tuvo sus representantes: por ejemplo, J. M. MarTínez VAL, "Polémica sobre la última encíclica”, Razón Española (1988) 191-199. 
a sus propios fallos. Sin embargo, Juan Pablo II conecta este análisis suyo con la constatación de la creciente interdependencia de nuestro mundo, un concepto que adelanta lo que poco después comenzará a llamarse globalización, al menos en sus aspectos sustanciales.

e) No faltan tampoco en SRS las menciones del colectivismo, pero hay que reconocer que su inserción en el texto resulta un tanto forzada y distorsiona el hilo general del discurso. Esencialmente se alude a él cuando se afirma que también atentan contra un verdadero desarrollo las privaciones sistemáticas de ciertas libertades y derechos fundamentales, entre las que se citan la libertad de iniciativa económica y la libertad religiosa [SRS 15, 33].

f) Juan Pablo II incorpora a la doctrina oficial de la Iglesia dos conceptos de interés, no sólo por su contenido sino también por las circunstancias que rodean su aceptación por el magisterio de la Iglesia. El primero es el de estructuras de pecado, concepto en el que muchos vieron una amenaza más para la vigencia del pecado personal y la conciencia del mismo: estas reservas explican también cómo SRS matiza las relaciones entre estructuras de pecado y pecado personal [SRS 36] ${ }^{10}$. El segundo concepto es el de solidaridad en un sentido más amplio que el clásico de la solidaridad entre los que comparten una misma situación oprimida: este contraste se ve entre LE (donde se habla de la solidaridad de la clase obrera [LE 8]) y SRS (donde la solidaridad se refiere al "otro distinto": solidaridad vertical y no sólo horizontal [SRS 39-40]) ${ }^{11}$.

\section{Encíclica conmemorativa de la primera encíclica social: Centesimus annus (1991)}

Con sólo observar la fecha de este documento se cae en la cuenta de que en el mundo se han producido cambios considerables: aunque todavía

10 Para toda esta cuestión puede verse:. W. EICHINGER, "Strukturen der Sünde. Zur theologischen Argumentation von Sollicitudo rei socialis", en: K. GABRIEL (Hrsg.), Die gesellschaftliche Verantwortung der Kirche, Patmos Verlag 1988, 117-136; J. R. FlechA, "La teología del desarrollo. Estructuras de pecado”, en: Comentarios a la Sollicitudo rei socialis, Acción Social Empresarial, Madrid 1990, 21-57.

11 La literatura sobre la solidaridad es abundante. Como botón de muestra: W. KLEIN, "Ein nenes Modell der Einheit. Die theologischeSicht der Menschheit als eine solidarische Gemeinschaft", en: K. GABRIEL (Hrsg.), l. c., 102-116; T. GofFI (1988), "Solidarietà, nome di pace e di progresso", Rivista di Teologia Morale n. 78, 53-57. 
recientes (apenas había transcurrido año y medio de la caída del muro de Berlín, cuando se hizo pública la CA), esos meses había sido suficientes para intuir el alcance de lo que había acontecido.

\subsection{Contexto y objetivos}

Hay que reconocer, sin embargo, que un análisis en profundidad de lo que 1989 significaba, hubiera exigido una distancia temporal mayor. No fue posible porque existía un compromiso previo a la caída del muro: la conmemoración del centenario de la primera encíclica social, la Rerum novarum de León XIII en 1891. La fecha de 1991 era, pues, inaplazable.

Se explica así que CA tiene un doble objetivo: "poner en evidencia la fecundidad de los principios expresados por León XIII", pero también "proponer el análisis de algunos acontecimientos de la historia reciente" [CA 3]. En realidad son dos objetivos estrechamente conectados: si la Doctrina Social de la Iglesia, cuyo centenario se conmemora, se ha desarrollado históricamente al hilo del propio desarrollo de los sistemas socioeconómicos, lo que había ocurrido en 1989 era sin duda un capítulo muy decisivo de esa historia (y para algunos el último ${ }^{12}$ ). Sin embargo, lo que aconteciera en 1989 no iba a afectar sólo al colectivismo, ni se podría interpretar - como hicieron algunos- como el triunfo definitivo del capitalismo y su consagración indiscutible. El golpe asestado al modelo colectivista con la caída del muro de Berlín es determinante, pero no se puede olvidar que en esos momentos el capitalismo también se enfrenta a una fuerte crisis de viabilidad. Esta crisis (más notoria allí donde más se ha desarrollado el Estado social) abona el resurgir de las tesis liberales y de propuestas de retorno al Estado mínimo, el modelo propio del liberalismo. Por tanto, 1989 si acaso sirve para agudizar más esta pregunta: si el colectivismo no es ya alternativa, ¿con qué tipo de capitalismo nos quedamos?

Esta cuestión está muy presente en toda la CA. En ella Juan Pablo II quiere analizar por qué cayó el colectivismo, pero más interesado está todavía por elaborar un juicio ético en profundidad sobre ese capitalismo que muchos presentan ya sin alternativas. Esta última cuestión sigue abierta, y en ella la

12 Es obligado citar aquí la obra de F. FunuYama (1992), El fin de la historia y el último hombre, Barcelona, Planeta, que responde a la coyuntura inmediatamente posterior a 1989. Una síntesis de esa obra: F. FunuYAma (1990), “El fin de la historia?”, Claves de la razón práctica n. 1, 85-96. 
Doctrina Social de la Iglesia está en debate con las corrientes neoliberales. Este debate conecta estrechamente con toda la tradición de la Doctrina Social de la Iglesia: porque, si es indudable que ésta excluyó desde siempre y sin ningún género de dudas el colectivismo marxista, no menos cierto es que también se mostró bastante crítica con el liberalismo, ya desde el siglo XIX. En cambio en la segunda mitad del siglo XX mostró una mayor sintonía con los modelos mixtos de organización económica, más cercanos a la socialdemocracia que al liberalismo clásico. La crisis de este modelo mixto en el Estado social ¿no le estaba obligando a revisar esta postura? En CA esta cuestión resultaba difícil de eludir.

\subsection{Contenido esencial}

A la hora de formular el contenido nuclear de CA seguiremos un camino distinto al de las dos encíclicas anteriores y procederemos en dos pasos. En primer lugar, se ofrecerá una síntesis de cada capítulo mostrando cómo el conjunto responde a una triple mirada: al pasado, al presente y al futuro (en términos de la misma encíclica: "una mirada retrospectiva", un "mirar alrededor", un "mirar al futuro" [CA 2]). En segundo lugar intentaremos sistematizar la respuesta de $\mathrm{CA}$ a la doble pregunta arriba formulada: ¿por qué cayó el colectivismo? ¿cómo enjuiciar éticamente al capitalismo?

El alcance de esa triple mirada permite mostrar todo el contenido de la encíclica capítulo por capítulo:

\section{Una mirada al pasado}

1. La Rerum novarum y la fecundidad de su doctrina a lo largo del siglo transcurrido [Capítulo 1: "Rasgos característicos de la Rerum novarum"]. Se sitúa la encíclica en su contexto histórico para hacer luego una "relectura" desde la problemática de hoy.

2. Principales acontecimientos del siglo transcurrido que confirman la doctrina de Rerum novarum [Capítulo 2: "Hacia las cosas nuevas de hoy"]. Entre ellos destacan: el fracaso del socialismo, tal como había sido previsto por León XIII; las transformaciones del Estado a partir del modelo liberal vigente en el siglo XIX; las dos guerras mundiales y las consecuencias de esta última con la división del mundo en dos bloques. Se subraya como la raíz de tantas tragedias es el abandono de la doctrina de la Iglesia sobre la verdadera libertad.

\section{RFS}


II. Una mirada al presente [Capítulo 3: "El año 1989"]

1. Las causas de la caída del colectivismo. La clave ha sido el recurso al diálogo y la solidaridad como alternativa a la lucha de clases. Dos factores explican la caída del colectivismo: la violación sistemática de los derechos del trabajador y la ineficacia de un sistema económico que viola derechos fundamentales (iniciativa, propiedad, libertad económica).

2. Las consecuencias que se siguen son de orden muy diverso: la Iglesia se reencuentra con el movimiento obrero; Europa se apresta a recorrer el camino hacia su unidad; pero la necesaria solidaridad con los países excomunistas no puede servir de excusa para olvidarse del tercer mundo.

\section{Una mirada al futuro}

1. El orden económico [Capítulo 4: "La propiedad privada y el destino universal de los bienes"].Partiendo dela doctrina sobre la propiedad, se pone de manifiesto la importancia creciente del ser humano en la economía. Esto exige revisar el modelo económico de mercado, aunque se acepte como instrumento de asignación de recursos, se rechaza de él la absolutización de la libertad económica que le acompaña; y se acepta también la libre empresa, con tal que sea entendida como una comunidad de personas.

2. El orden político [Capítulo 5: "Estado y cultura"]. Se discute el alcance de una verdadera democracia que respete la subordinación de la libertad humana a la verdad, y se concretan las competencias del Estado en el orden económico, saliendo al paso de los excesos intervencionistas del Estado de bienestar.

3. El orden cultural [continúa el Capítulo 5]. Se parte de las culturas de los pueblos (como "patrimonio de los valores heredados y adquiridos") y de su inevitable evolución, para hablar luego de la cultura como fenómeno universal: una cultura de la paz, a cuya consolidación la Iglesia quiere prestar una eficaz contribución.

IV. Síntesis conclusiva [Capítulo 6: "El hombre es el camino de la Iglesia"]. Como el título del capítulo sugiere, el centro de la Doctrina Social no es otro que la visión del hombre: así debe ser divulgada y llevada a la acción. 


\subsection{Las grandes preguntas pendientes}

Con este esquema del contenido como fondo, veamos ahora cómo da respuesta CA a las dos preguntas arriba formuladas. Dicha respuesta no está sistemáticamente desarrollada en el texto: hay que reconstruirla recogiendo pasajes diferentes que, puestos en relación, ofrecen una propuesta bastante coherente en sí y muy en sintonía con posiciones encontradas en las dos encíclicas anteriores.

Para elaborar dicha respuesta hay que tener en cuenta dos cosas. En primer lugar, que la respuesta a una y otra pregunta se hace siguiendo el mismo método. Segundo, que este método consiste en considerar capitalismo y colectivismo como dos modelos globales de organización de la sociedad, distinguiendo en cada uno de ellos un triple nivel: económico, político y ético-cultural (ya en el punto III del esquema anterior se observaba este modelo de análisis $)^{13}$.

Permítasenos identificar en primer lugar cuáles son los tres sistemas que constituyen uno y otro modelo de organización social. Para ello nos valemos del cuadro que sigue:

\begin{tabular}{|l|c|c|}
\cline { 2 - 3 } \multicolumn{1}{c|}{} & Capitalismo & Colectivismo \\
\hline Sistema económico & Economía de mercado & Planificación central \\
\hline Sistema político & Democracia pluralista & $\begin{array}{c}\text { Autoritarismo de } \\
\text { partido único }\end{array}$ \\
\hline Sistema ético-cultural & Liberalismo & Marxismo \\
\hline
\end{tabular}

¿Por qué fracasó el colectivismo? La respuesta de CA es relativamente sencilla. Parte de dos afirmaciones: $1^{\underline{a}}$ ) el marxismo tiene una concepción de la persona humana muy negativa, la considera incapaz de tomar decisiones libres y termina diluyéndola en la sociedad y reduciéndola a una pieza de esa gran maquinaria social; $2^{\mathfrak{a}}$ ) tal antropología no puede conducir más que

13 Este modelo de análisis social lo encontramos hoy en autores de tendencias muy diferentes: desde los neoconservadores y neoliberales norteamericanos hasta los neomarxistas de la Escuela de Frankfurt. 
a un sistema económico y a un sistema político que sustituya la libertad de elección y la iniciativa de la persona por un modelo centralizado de decisiones (planificación económica y totalitarismo político) [CA 13, 25, 44]. De estas dos premisas se sigue una conclusión fáctica: los seres humanos no pueden soportar a la larga un régimen así, contra el que terminarán por rebelarse. Eso es lo que han demostrado los hechos en $1989^{14}$.

¿Cómo juzgar éticamente al capitalismo? La respuesta a esta pregunta es más compleja, pero tiene como base igualmente la interacción de los tres sistemas. La postura de CA, que está dispersa en diferentes pasajes, se sintetiza así: lo cuestionable del capitalismo no es su sistema económico o su sistema político, sino su sistema ético-cultural. Veámoslo por partes:

- La economía de mercado - en cuanto economía caracterizada por la iniciativa privada, la libertad de empresa y la propiedad privada- no ofrece, en principio, ninguna dificultad moral. Pero sí la concepción de la libertad que la anima: una libertad sin restricciones en lo económico, que se convierte de hecho en un obstáculo para la libertad integral de muchos [CA 42, cf. también 39]. La forma de entender la libertad corresponde, evidentemente, al sistema ético-cultural.

- Tampoco la democracia pluralista —en cuanto sistema de participación de los ciudadanos y de control de los gobernantes-plantea en sí misma problemas éticos. Los problemas vienen, nuevamente, de la concepción de la libertad que subyace en este caso: una libertad sin límites (la voz de la mayoría), que no reconoce la sumisión a una verdad objetiva de orden moral, que está por encima de ella [CA 46]. Estamos de nuevo con dificultades que provienen del sistema ético-cultural.

Como se ve, es en la concepción de la libertad donde tropezamos siempre: ya ocurrió con el colectivismo, y ahora ocurre con el capitalismo. En todos los casos, Juan Pablo II pone como contrapunto la visión cristiana de la libertad, que se convierte así en eje de toda la encíclica: una libertad que se reconoce como esencial al ser humano (contra el marxismo), pero una libertad que debe ser entendida de forma integral (no reducida a libertad económica o libertad en el mercado) y sometida a una verdad moral objetiva que le pone

14 Evidentemente las premisas de esta argumentación no serían admitidas por el marxismo heterodoxo, que venía distanciándose inequívocamente de la ortodoxia soviética. Pero, puestas las premisas y visto lo ocurrido en los países comunistas, es difícil negar la argumentación aplicada al llamado "socialismo real". 
límites. Es cierto que Juan Pablo II no menciona nunca el liberalismo en esta parte de su encíclica, pero creemos que no es incorrecto identificarlo sin rodeos como la ideología inspiradora o el sistema ético-cultural del capitalismo, que el papa critica ${ }^{15}$.

\subsection{Puntos a destacar y principales aportaciones}

Esta más extensa exposición de los contenidos esenciales de CA permite ahora destacar las aportaciones más significativas de éste su último gran documento social:

a) Se confirma que la aportación esencial de la Doctrina Social de la Iglesia se localiza en el nivel de los valores y de la antropología que les da base. Desde una visión cristiana de la persona Juan Pablo II toma posición clara ante los modelos de organización de la sociedad, tanto ante el ya casi desaparecido colectivismo, como ante el capitalismo dominante.

b) Pero, en relación con las dos encíclicas anteriores, CA avanza en el modelo de análisis incorporando la dimensión política: resulta así un modelo que relaciona lo económico con lo político y lo ético-cultural. Ahora bien, el sistema político, no sólo es el más someramente analizado, sino el más deficientemente interpretado. Falta, en nuestra opinión, una comprensión más adecuada de lo que significa la democracia en una sociedad éticamente plural, que nunca busca definir la verdad, sino más modestamente establecer unas normas para la convivencia [CA 46]. Cuál es el alcance de unos principios morales universales en una sociedad plural y cómo acceder a ellos son cuestiones muy complejas y delicadas, que CA no aborda con la profundidad deseada.

c) CA muestra, al compararla con LE y SRS, con particular evidencia la dimensión histórica de la Doctrina Social de la Iglesia. Lo ocurrido en 1989 es de tales dimensiones que ha sido inevitable, en ese corto periodo de tiempo, proceder a replanteamientos considerables. Afectan precisamente, a las posiciones mantenidas frente a los dos sistemas.

15 En CA 42 habla de "una ideología radical de tipo capitalista, que rechaza incluso el tomarlos en consideración [se refiere a los fenómenos de marginación y explotación, que perviven en el mundo después de la caída del colectivismo], porque a priori considera condenado al fracaso todo intento de afrontarlos y, de forma fideísta, confía su solución al libre desarrollo de las fuerzas de mercado". 
d) Si en LE Juan Pablo II parecía admitir ciertas posibilidades de regeneración del colectivismo siempre que admitiera incorporar mayores cotas de participación, el CA parece convencida de que ello era imposible. Es lo que se deduce de su razonamiento: la concepción marxista de la persona no permite esperar que ésta sea digna de la confianza que exigiría una mayor participación en la vida económica y política ${ }^{16}$.

e) Con el capitalismo, la postura de la CA gana en realismo y, al mismo tiempo, en rigor analítico. El realismo se manifiesta en que comprende que es, hoy por hoy, el único sistema existente, y sería poco esclarecedor hacer descalificaciones generales. El rigor analítico se constata, no sólo en los datos que aportamos más arriba, sino también en el hecho de admitir varias formas de capitalismo. Aquí Juan Pablo II se sitúa en continuidad con los documentos de Juan XXIII y Pablo VI y se inclina por un capitalismo mixto, que recuerda al construido por la socialdemocracia y la democracia cristiana [CA 19]; pero con la novedad de mostrarse crítico al mismo tiempo frente a los excesos de intervención del Estado en dicho modelo [CA 48].

f) Estas prevenciones sobre el excesivo estatalismo han hecho pensar a algunos en una sintonía de fondo entre Juan Pablo II y la mentalidad neoliberal, viendo en ello el acercamiento de la Doctrina Social de la Iglesia al liberalismo, tantas veces deseado desde ambientes diferentes ${ }^{17}$. Es cierto que Juan Pablo II no hace una crítica directa y explícita al (neo)liberalismo, designándolo por su nombre. Ahora bien, una lectura desapasionada de la encíclica permite descubrir en ella elementos para una crítica muy contundente del pensamiento liberal, y concretamente en sus versiones más actuales (neoliberales). Evidentemente hoy nadie discute ya la eficacia del mercado: en ese

16 Sin embargo falta una mayor precisión en la distinción entre colectivismo y marxismo. Juan Pablo II, que había sido tan riguroso en el uso de los términos en sus dos anteriores encíclicas, utiliza ahora indistintamente colectivismo, comunismo, socialismo, socialismo real y marxismo. Especialmente problemática es la confusión entre colectivismo y marxismo, sobre todo cuando se habla de fracaso del marxismo, donde sólo convendría hablar estrictamente de fracaso del colectivismo [CA 23, 27].

17 Esto ya ocurrió desde el momento mismo de la publicación de la encíclica. El Wall Street Journal de 4 de mayo de 1991 se alegraba de ver cómo el papa aceptaba, por fin, que el capitalismo es "el corolario económico de la comprensión cristiana de la naturaleza y del destino del hombre" (citado por J. I. González Faus (2005), Para comprender a Karol Wojtyla, Santander, Sal Terrae, 69, nota 23). 
sentido el debate entre las alternativas capitalismo-colectivismo ha perdido todo su interés. Pero la aceptación del mercado exige ser cautos frente a una desmedida exaltación del mismo como el mejor sistema para favorecer la libertad. Se olvidarían entonces los perjuicios que se siguen de esa afirmación de la libertad sin reservas, en una economía entregada al mercado sin restricciones ${ }^{18}$. ¿O es que las últimas dos décadas no ofrecen pruebas palmarias de que la liberalización de los mercados incrementa la producción, pero al precio de un incremento paralelo de las desigualdades? ${ }^{19}$.

g) La solución última a la que apunta CA parece ser la de una progresiva sustitución del sistema ético-cultural del capitalismo por una concepción más aceptable de la libertad (la que deriva de la tradición cristiana). Vemos de nuevo cómo Juan Pablo II pone su centro de atención en el sistema ético-cultural o sistema de valores. Aunque no es pensable una sustitución casi mecánica de un sistema ético-cultural por otro, cabe aceptar que ésa será la tensión que el cristiano siempre estará invitado a mantener: entre conceptos insuficientes de libertad y otro que haga más justicia a una concepción integral de la persona. Pero cabe también discutir si es pensable desvincular el sistema ético-cultural del sistema económico y del político en el capitalismo: ¿no existe una relación muy estrecha entre los tres sistemas, de forma que recíprocamente se refuerzan? ${ }^{20}$

h) Por último hay que decir una palabra, también a propósito de CA, sobre el análisis social que realiza el papa en ella. Ante todo, hay que reconocer

18 Cf. J.-Y. Calvez (1991), “Centesimus annus et le libéralisme”, Études 375, 625-632.

19 Esta línea sigue abierta hoy. Como expresión reciente de este deseo de aproximación puede verse: M. NovAK, "The Social Thought of Pope John Paul II", Chicago Studies 29 (2000) 144-161. El autor insinúa constantemente cómo el pensamiento de Juan Pablo II coincide con los presupuestos del liberalismo: la libertad humana como creatividad, las reservas respecto al papel del Estado, etc. Pero ignora las críticas que $\mathrm{CA}$ hace al concepto de libertad del capitalismo. Ignora igualmente cuántas veces recurre Juan Pablo II para ilustrar su posición a la doctrina de León XIII sobre la libertad, que fue elaborada precisamente para oponerse al liberalismo de su tiempo. Por último - y es muy indicativo del distinto enfoque del pensamiento social cristiano y del liberalismo-, Novak repite una y otra vez que la justicia social es sólo una virtud personal, un hábito de los individuos.

20 Véase esta crítica en: CL. Boff (1991), "O Capitalismo triunfante na visão atual de João Paulo II (Lectura da Centesimus annus a partir do Terceiro Mundo)", Revista Eclesiastica Brasileira $51,825-846$. 
que la cercanía de los hechos es tanta que falta una mayor perspectiva temporal, siempre deseable cuando se trata de acontecimientos de tanta envergadura. Por otra parte, en el análisis del proceso parece que se contempla muy en primer plano lo que sucedió en Polonia (la resistencia de la clase obrera, el papel de la Iglesia [CA 23-25]), que no puede hacerse extensivo a lo que sucedió en otros países del entorno. En todo caso, Juan Pablo II reconoce que este tipo de análisis no posee la misma autoridad que las afirmaciones doctrinales, aunque no por eso debe ser excluido de un documento que quiere analizar éticamente los hechos sociales ${ }^{21}$.

\section{Reflexiones de conjunto}

Después de este recorrido por las tres grandes encíclicas sociales de Juan Pablo II parece conveniente sistematizar algunas reflexiones que marquen las coordenadas de su pensamiento social. Con ello no queremos hacer una síntesis completa del mismo, sino sólo destacar algunos puntos que derivan del recorrido precedente.

\subsection{Encíclicas sociales y preocupaciones de un pontificado}

Juan Pablo II dedicó a temas sociales tres de las catorce encíclicas que publicó a lo largo de su pontificado. Y las tres se sitúan en la primera mitad del mismo. ¿Es esto una casualidad? ¿Obedece más bien a razones específicas?

Algunos autores relacionan este hecho con una cierta evolución de las preocupaciones dominantes en los años de pontificado. Porque el final de los años 1980 constituye un hito histórico, no sólo por la caída del colectivismo, sino también por el fracaso electoral del sandinismo en Nicaragua. Ambos

21 Es de interés transcribir este pasaje donde queda clara la distinción entre afirmaciones doctrinales y conclusiones del análisis social [CA 3]: "La presente encíclica trata de poner en evidencia la fecundidad de los principios expresados por el papa León XIII, los cuales pertenecen al patrimonio doctrinal de la Iglesia y, por ello, implican la autoridad del magisterio. Pero la solicitud pastoral me ha movido además a proponer el análisis de algunos acontecimientos de la historia reciente. Es superfluo subrayar que la consideración atenta del curso de los acontecimientos, para discernir las nuevas exigencias de la evangelización, forma parte del deber de los pastores. Tal examen, sin embargo, no pretende dar juicios definitivos, ya que de por sí no atañe al ámbito específico del magisterio". 
hechos supusieron un golpe contundente para los movimientos revolucionarios y las ideologías que los alimentaban, en las cuales veía Juan Pablo II una amenaza para la Iglesia.

Esto tiene que ver con su postura respecto a la teología de la liberación, que en la primera etapa de su pontificado fue objeto de un tratamiento minucioso y estudiado: aunque nunca se llegó a condenarle ni se expresó un rechazo abierto contra ella, no faltaron las puntualizaciones y los matices en torno al concepto mismo de liberación. Recuérdense los dos documentos sobre el tema, de la Congregación para la Doctrina de la Fe, donde se pudo observar una suavización de las posturas del segundo respecto al primero $\mathrm{y}$ un indisimulado esfuerzo por integrar en lo posible diversos elementos de esa corriente teológica dentro de la doctrina oficial ${ }^{22}$.

Lo que preocupaba a Juan Pablo II de la teología de la liberación era el peligro que ella implicaba para una secularización progresiva de la Iglesia. Por eso el Papa procurará relacionar esa liberación de toda situación opresiva en este mundo con aquella otra liberación de las raíces profundas de todo mal, que es el pecado, liberación que sólo se consumará más allá de la existencia terrena.

Esta preocupación se refleja también en las encíclicas sociales, aunque no tenga un excesivo peso específico. Sólo SRS dedica un número entero al tema [SRS 46]. Aludiendo expresamente a ella, se critica la reducción del desarrollo a lo económico, ignorando la dimensión cultural, trascendente y religiosa del ser humano, y se afirma que el principal obstáculo para la liberación es el pecado y las estructuras que llevan al mismo ${ }^{23}$.

Esta preocupación más de fondo por la secularización de la Iglesia se mantiene a lo largo de sus 26 años en la sede romana y está detrás igualmente de lo que son sus nuevos centros de atención desde los años 1990: el diálogo ecuménico e interreligioso ${ }^{24}$. Porque en un mundo tan secularizado,

22 Congregación para la Doctrina de la Fe (1984), Libertatis nuntius. Instrucción sobre algunos aspectos de la teología de la liberación; CONGREGACIÓN PARA LA DoctRINA DE LA Fe (1986), Libertatis conscientia. Instrucción sobre libertad humana y liberación.

23 M. AlCALÁ, "La encíclica Sollicitudo rei socialis y las teologías de la liberación", Revista de Fomento Social 43 (1988) 461-471, ve algunos puntos de contacto de SRS con la teología de la liberación, tanto metodológicos (la preferencia por un método inductivo) como de contenidos (dependencia, opción por los pobres y, sobre todo, las estructuras de pecado).

24 Cf. J. B. LiBÂnIo (2005), “Juan Pablo II: una mirada retrospectiva”, Sal Terrae 93, 457-470. 
los creyentes, cualquiera que sea su credo, tienen que aunar fuerzas para mantener viva la dimensión trascendente y religiosa de la existencia. En esto, además, Juan Pablo II no puede ignorar su experiencia de Polonia, donde la Iglesia ha desempeñado un papel decisivo para mantener la identidad de un pueblo al que la historia de los últimos siglos tanto maltrató ${ }^{25}$.

\subsection{Confirmar y clarificar el concepto de Doctrina Social de la Iglesia}

Esta es una aportación indudable de Juan Pablo II, que comenzó su pontificado con un ambiente de serias reservas respecto al término mismo de "Doctrina Social de la Iglesia". En efecto, algunos lo consideraban como un producto de la mentalidad anterior al Vaticano II, y tributario de aquella forma de entender las relaciones entre la Iglesia y la sociedad que el mismo Concilio había puesto en entredicho. No puede negarse que Pablo VI contribuyó a dar un giro al pensamiento social de la Iglesia abriéndolo a los nuevos problemas del momento y adaptándolo a las nuevas coordenadas del cristiano en la sociedad moderna ${ }^{26}$. Pero él mismo evitaba el término "Doctrina social", como hiciera ya el Concilio, sustituyéndolo por otros de carácter más flexible (como "enseñanzas sociales de la Iglesia", por ejemplo).

Todas estas posibles vacilaciones quedaron superadas desde una de las primeras intervenciones solemnes de Juan Pablo II: su discurso en la inauguración de la III Conferencia General de Episcopado Latinoamericano en Puebla (28 enero 1979). En él concluye confiando a los obispos del continente la tarea pastoral "de sensibilizar a los fieles acerca de esta doctrina social de la Iglesia", de la que afirma: "nace, a la luz de la Palabra de Dios y del magisterio auténtico, de la presencia de los cristianos en el seno de situaciones cambiantes del mundo, en contacto con los desafíos que de ésas provienen"27.

Pero más importante que reafirmar el valor y la validez de la Doctrina Social de la Iglesia es su contribución en torno al contenido mismo que hay que dar al concepto. Eso lo hace con dos precisiones que nos parecen funda-

25 Sobre el influjo de la historia de Polonia en Juan Pablo II puede verse: J. I. GonzÁlez Faus, l.c..

26 A ello responden, respectivamente, la encíclica Populorum progressio (1967) y la carta Octogesima adveniens (1971).

27 Parte III, número 7. 
mentales: la Doctrina Social de la Iglesia es teología moral y es instrumento de la evangelización.

La Doctrina Social es teología moral. No es sólo una versión en cristiano de las ciencias sociales (en el sentido en que se hablaba antes de "sociología cristiana", por ejemplo). Porque su punto de partida y la fuente primera en que se inspira es la revelación: lo que la Sagrada Escritura y la tradición de la Iglesia nos dicen sobre lo que es la persona humana y la sociedad. Lo que hay que buscar, por tanto, en ella no son modelos alternativos a los vigentes (capitalismo o socialismo), como en otros tiempos se pretendiera, sino sólo grandes orientaciones para criticar los actuales o iluminar la búsqueda de otros mejores. Desde aquí se entiende su insistencia - como vimos tanto en SRS como en CA- de trascender el análisis de los sistemas sociales para llegar a los sistemas de valores que les sirven de sustento: es ahí donde se mueve la Doctrina Social de la Iglesia. Pero — iojo! — esto no significa que los cristianos no deban comprometerse en la búsqueda de modelos alternativos: sólo que esos modelos nunca se podrán considerar como inequívocamente derivados de la Doctrina Social de la Iglesia.

La Doctrina Social es instrumento de evangelización. La Doctrina Social no es un saber especializado, propio de algunos grupos reducidos de creyentes con una vocación específica, sino que conecta estrechamente con la misión misma de la Iglesia y con la vocación de cada uno de sus miembros. Si el Vaticano II elaboró una doctrina de la Iglesia que hacía de la misión evangelizadora su razón de ser más profunda ${ }^{28}$, Juan Pablo II se encarga ahora de colocar a la Doctrina Social en conexión directa con esa misión. Eso sí, es preciso incorporar aquí un concepto de evangelización adecuado, tal como hizo Pablo VI en continuidad con el Vaticano II: como un proceso complejo, que se inicia con el testimonio de vida (que lleva al compromiso de transformar la realidad social) y que sólo en un momento ulterior, y si se dan las condiciones para ello, aborda el anuncio explícito de Jesús ${ }^{29}$.

28 Este es un punto crucial de la teología contemporánea, que marca toda la relación de la Iglesia con la sociedad hoy. Puede verse: I. Cамасно (1998), Doctrina Social de la Iglesia. Una aproximación histórica, Madrid, San Pablo, capítulo 10.

29 Cf. lo dicho supra en la nota 7. Esta doctrina de Pablo VI es esencial para comprender en sus verdaderas coordenadas todo el discurso posterior de Juan Pablo II sobre la evangelización y sobre la nueva evangelización. 


\subsection{El discurso ético de la Doctrina Social de la Iglesia}

Decir que la Doctrina Social forma parte de la teología moral comporta tomar cierta distancia de la tradición iusnaturalista que tradicionalmente la marcó. Este cambio de orientación es también muy característico de Juan Pablo II, y quedó ya patente en la primera de sus encíclicas, con la que inauguró su pontificado: Redemptor hominis (4 marzo 1979). Quedó expresado en aquella fórmula, no siempre de fácil comprensión: "El hombre es el camino para la Iglesia" 30 . La realidad humana, según el designio de Dios, es la que la Iglesia está llamada a anunciar siempre y en primer lugar.

Esto no quiere decir que Juan Pablo II emplee siempre un lenguaje estrictamente teológico. Él es consciente de que el mundo plural y secular de hoy no entiende muchas veces ese lenguaje y entonces es preciso recurrir a otro basado sólo en la racionalidad común a todos los seres humanos. Es entonces cuando recurre a la ley natural, a pesar de que los escollos con que ha tropezado este enfoque a lo largo de siglos se acrecientan hoy, no sólo por la distancia entre dicho concepto y la mentalidad actual, sino sobre todo por la complejidad de los temas a que el Papa los ha aplicado (concretamente, los relativos a la ética personal y sexual y a la bioética).

Pero tampoco Juan Pablo II recurre siempre a un razonamiento deductivo, en coherencia con el iusnaturalismo. No falta en sus escritos un enfoque más inductivo que le hace partir del análisis de la realidad y de la reflexión sobre ella: SRS es, de las tres encíclicas estudiadas, la que más claramente practica ese método; pero tampoco está ausente en CA. Por este camino Juan Pablo II se aventura a veces en interpretaciones audaces de los hechos, que han suscitado no poca polémica: ahí están para probarlo la explicación que da de las causas del conflicto Norte-Sur en SRS o de la caída del colectivismo en CA, o el modelo de análisis que aplica a capitalismo y colectivismo en esta última encíclica. Como ya quedó indicado más arriba, él mismo reconoce que el contenido de estos análisis no tiene el valor de las afirmaciones propiamente doctrinales.

30 JuAN PABLo II, Redemptor hominis n. 21. Véase esta otra formulación, algo más explícita: "El hombre en la plena verdad de su existencia, de su ser personal y a la vez de su ser comunitario y social -en el ámbito de la propia familia, en el ámbito de la sociedad y de contextos tan diversos, en el ámbito de la propia nación, o pueblo (y posiblemente sólo aún del clan o tribu), en el ámbito de toda la humanidad - este hombre es el primer camino que la Iglesia debe recorrer en el cumplimiento de su misión, él es el camino primero y fundamental de la Iglesia, camino trazado por Cristo mismo, vía que inmutablemente conduce a través del misterio de la Encarnación y de la Redención" (n. 14). 
En resumen, Juan Pablo II armoniza tres niveles de discurso: el teológico (que considera el más genuino de la Doctrina Social de la Iglesia), el ético-filosófico (más apto para el diálogo en la sociedad actual) y el del análisis social (que es previo a la reflexión ética).

\subsection{Globalización y solidaridad}

El hecho, ya señalado, de que las tres grandes encíclicas sociales de Juan Pablo II se localicen en la primera mitad de su pontificado permite comprender el escaso lugar que ocupa en ellas la globalización. Sin embargo se encuentran algunos pasajes que merecen ser destacados.

En CA encontramos una advertencia que tiene que ver con la integración de los mercados. Se dice que los países más pobres no deben buscar la solución aislándose del mercado mundial: por el contrario, "el mayor problema está en conseguir un acceso equitativo al mercado internacional” [CA 33]. Sabemos que esto no es fácil en el mundo globalizado y sometido a la ley de los más fuertes, pero no se debe renunciar a conseguirlo.

Pero es en SRS donde encontramos una contribución verdaderamente innovadora y de alcance. En esta encíclica Juan Pablo II se adelantó con una reflexión ética de gran interés para la globalización al establecer una estrecha conexión entre interdependencia y solidaridad [SRS 38]. Probablemente si Juan Pablo II hubiera escrito SRS diez años después hubiera hablado de globalización en lugar de interdependencia. Porque ¿qué es la globalización sino, en primer lugar, interdependencia entre las economías? Juan Pablo II razona de forma inductiva: si tomamos como un hecho irreversible el proceso globalizador, no hay otra respuesta éticamente aceptable que el asumir esa interdependencia responsabilizándonos todos de todos (y eso es la solidaridad) ${ }^{31}$.

Las aplicaciones de este principio son innumerables y sirven de contrapeso a una economía basada sólo en la competencia y en la lucha entre iguales. Si competir es luchar, aunque sea en una lucha limpia (cosa que no siempre ocurre), la solidaridad es cooperación. Competir y cooperar son dinámicas complementarias, y en un mundo tan competitivo es urgente recordar este

31 Cf. J. L. MARTínez (2005), "El personalismo solidario de Juan Pablo II. Convertir la interdependencia en solidaridad", Sal Terrae 93, 437-456; M. P. SiclidanI (2004), "La globalizzazione nel magistero de Giovanni Paolo II", Apollinaris 77, 667-677. 
complemento de la solidaridad como indispensable.

Ahora bien, la globalización no es sólo interdependencia económica: tiene también aspectos políticos y culturales. De los políticos se ocupa menos el magisterio de Juan Pablo II, y mucho más de los económicos y culturales. Permítasenos citar dos textos relativos a unos y otros.

La crítica más elaborada a una globalización económica entendida sólo como mercado está en el documento que siguió al Sínodo de América. Se resume así lo positivo y lo negativo de la globalización:

Desde el punto de vista ético, [la globalización] puede tener una valoración positiva o negativa. En realidad, hay una globalización económica que trae consigo ciertas consecuencias positivas, como el fomento de la eficiencia y el incremento de la producción, y que, con el desarrollo de las relaciones entre los diversos países en lo económico, puede fortalecer el proceso de unidad de los pueblos y realizar mejor el servicio a la familia humana. Sin embargo, si la globalización se rige por las meras leyes del mercado aplicadas según las conveniencias de los poderosos, lleva a consecuencias negativas. Tales son, por ejemplo, la atribución de un valor absoluto a la economía, el desempleo, la disminución y el deterioro de ciertos servicios públicos, la destrucción del ambiente y de la naturaleza, el aumento de las diferencias entre ricos y pobres, y la competencia injusta que coloca a las naciones pobres en una situación de inferioridad cada vez más acentuada. La Iglesia, aunque reconoce los valores positivos que la globalización comporta, mira con inquietud los aspectos negativos derivados de ella ${ }^{32}$.

La globalización entendida desde la competencia y el individualismo resultará mortífera para los más débiles. No cabe resignación ante un proceso considerado imparable, sino que es preciso conducirlo en función de los intereses de todos, y en especial de los menos agraciados por ella.

En cuanto a la globalización cultural, véase este texto que responde a aquel escepticismo que mostrábamos más arriba ante la propuesta de cambiar el sistema ético-cultural del capitalismo en CA:

Una de las preocupaciones de la Iglesia acerca de la globalización es que se ha convertido rápidamente en un fenómeno cultural. El mercado como mecanismo de intercambio se ha convertido en instrumento de una nueva cultura. Muchos observadores han reconocido el carácter intrusivo, incluso invasor, de la lógica del mercado, que va reduciendo cada vez más el área que queda a la comunidad humana para la acción pública y voluntaria a todos los niveles. El mercado impone su modo de pensar y de actuar e imprime su escala de valores en el comportamiento humano ${ }^{33}$.

32 Juan Pablo II (1999), Exhortación apostólica Ecclesia in America, n. 20.

33 Juan Pablo II (2001), Discurso a la Academia Pontificia de Ciencias Sociales, n. 3 
En aquella misma ocasión daba la clave que debía orientar todo el proceso globalizador: el desarrollo de la persona, a cuyo servicio han de estar todas las instituciones sociales, evitando una "globalización en la marginación" y persiguiendo una "globalización en la solidaridad". Esta última cita puede ser una buena síntesis de todo el pensamiento social de Juan Pablo II y es útil para concluir:

Ningún sistema puede ser fin en sí mismo, y es necesario insistir en el hecho de que la globalización, como cualquier otro sistema, debe estar al servicio de la persona, de la solidaridad y del bien común ${ }^{34}$.

\section{Conclusión}

No hemos pretendido hacer una síntesis de todo el pensamiento social de Juan Pablo II. Nos hemos limitado prácticamente a sus tres grandes encíclicas. Y en ellas hemos destacado lo que nos han parecido los hilos conductores de su pensamiento. Hemos prescindido, en cambio, de otros temas puntuales que hubieran alargado excesivamente este trabajo. Pero ello creemos que ha sido suficiente para comprobar que su contribución ha sido valiosa y original.

No puede ignorarse que la figura de Juan Pablo II ha sido controvertida. Pero probablemente es en las cuestiones sociales donde sus ideas han resultado más audaces, hasta el punto de suscitar un mal disimulado rechazo en ambientes que se enorgullecen de su fidelidad al magisterio de la Iglesia.

Los años que le han tocado vivir han estado marcados por hechos de gran alcance, como han sido la caída del muro de Berlín, el avance de la globalización o la secularización que parece invadir a todas las sociedades. En ese marco ha destacado la personalidad arrolladora del pontífice recientemente fallecido, el cual, desde la experiencia particular de su país natal y de su historia, ha querido reforzar la presencia de la Iglesia en la sociedad del tránsito hacia un nuevo siglo ${ }^{35}$. Habremos de dejar que el paso de tiempo se encargue de hacer un juicio más objetivo de la figura de Juan Pablo II después de este largo pontificado.

34 Ibid., n. 2.

35 Cf. V. Conzemius (2005), “Juan Pablo II: el valedor de la justicia en el mundo”, Sal Terrae 93, 501-519. 